\title{
Editorial
}

\section{LESSONS LEARNED FROM THE TEACHERS OF THE PAST: A GREAT FUTURE BEHIND US}

At the end of the 1970s, correct ACL reconstruction and treatment of other knee ligament injuries were the new frontier for modern sports medicine - the Eldorado, the land to be conquered. Now, after a journey lasting more than 40 years, which has seen thousands of studies, papers, ideas, proposals and different surgical techniques, we are (even though we have yet to hit the center of the target) very close to the final goal. In the course of this journey, the innovative and brilliant intuitions of some teachers have been temporarily forgotten and abandoned, before subsequently being re-evaluated, reconsidered, partially corrected, and shown to be valid.

The lesson imparted by Prof. Perugia, described in this issue by one of his more brilliant alumni, is one that needs to be understood and taken on board by the pioneers of the new frontier of sports medicine in the $21^{\text {st }}$ century, that of biotech solutions.

The papers in this issue of JOINTS cover a range of topics - fresh frozen allografts for shoulder reconstructions, polyurethane meniscal scaffolds, growth factors for muscle lesions, microfractures combined with PRP - and they provide a small but significant sample of the current trends in modern sports medicine literature.

How much enthusiasm, how much energy, how many ideas, and how many dedicated new procedures need to be developed in order to repair a chondral lesion and try to reproduce the characteristics of native cartilage using biotech solutions?

And how many doubts and unanswered questions remain?

Is biological joint replacement using osteochondral bipolar fresh allografts a reliable and durable solution, or are significant advances, in knowledge and technique, still needed?

What is the basis for the excellent clinical and functional results of meniscal scaffolds, given that new meniscal tissue ingrowth similar to the original is yet to be achieved?

Like a modern-day sorcerer's apprentice, we look at test tubes of PRP without knowing exactly how to manage these cells, and without fully understanding their positive characteristics and disadvantages, how concentrated they need to be in order to be really effective, or how long their efficacy will last.

At the same time we are aware that traditional surgery alone has gone as far as it can, and that without effective development and improvement of biotechnologies, it will not allow us to get close to the brilliant results we strive for.

Is it really likely that, in 20 years' time, we will still be treating and repairing chondral and/or ostechondral lesions with a simple microfracture technique? This is like asking, at the end of the 1970s, whether an ACL tear could go on being repaired simply by reinserting acutely the stump of the ligament in its anatomical area of insertion.

In the light of these considerations, the lessons of Prof. Perugia and other teachers clearly take on much more meaning.

How many intuitions and new proposals in the field of biotech solutions will stand the test of time? How many of them will allow us to reach our Eldorado?

This is a difficult question to answer at the present time, but as the evolution of ACL surgery has shown us, nothing (apart from the truly superfluous and useless) should be discarded or forgotten.

Who, when ACL reconstructions were being performed using only the central third of the patellar tendon as graft, would ever have imagined reconstruction with the semitendinosus (plus the gracilis according to Prof. Perugia's intuition) making the strong comeback that, with improvements and corrections, it has done over the past 15 years?

Who, when the ACL was considered the only cause of rotational instability of the knee, would have imagined that the "periphery" would once again be considered as important as, or more important than, the central pivot?

Very often our future lies in our own hands, in our capacity to dream and imagine. But very often, to find it, we need to look back over our shoulders at what has gone before. To go on evolving and advancing it is sometimes necessary to re-evaluate, rethink and reinvent what has been done in the past.

Massimo Berruto Associate Editor

Joints 